\title{
Coverings of Convex Sets and Volume Inequalities
}

\author{
B. Carl $^{1}$ and D. Edmunds ${ }^{2}$ \\ ${ }^{1}$ Fakultät für Mathematik und Informatik, Universität Jena, \\ D-07740 Jena, Germany \\ carl@minet.uni-jena.de \\ ${ }^{2}$ School of Mathematical Sciences, University of Sussex, \\ Brighton, Sussex BN1 9QH, England \\ d.e.edmunds@sussex.ac.uk
}

\begin{abstract}
We give diverse coverings of convex sets and sections of convex sets in $R^{n}$ by balls and cylinder sets, and establish volume inequalities which generalize the famous Santalo and inverse Santalo inequalities. The tools which we use are developed from the viewpoint of geometric and functional analysis.
\end{abstract}

\section{Survey of Some of the Main Geometrical Results}

To state the main geometric results we recall some standard notation. A convex, symmetric, compact subset $B$ of $\mathbf{R}^{n}$ with the origin as an interior point will be called a ball. The polar ball $B^{\circ}$ of $B$ is defined by

$$
B^{o}:=\left\{a \in \mathbf{R}^{n}:\langle x, a\rangle \leq 1 \text { for all } x \in B\right\} .
$$

Moreover, we use the term "ellipsoid" to mean any ball of the form

$$
\mathcal{E}=T\left(B_{l_{2}^{n}}\right),
$$

where $T$ is a linear isomorphism on $\mathbf{R}^{n}$ and $B_{l_{2}^{n}}$ stands for the Euclidean unit ball of $\mathbf{R}^{n}, l_{2}^{n}$ being $\mathbf{R}^{n}$ equipped with the Euclidean norm. Furthermore, the polar $\mathcal{E}^{\text {o }}$ of the ellipsoid $\mathcal{E}$ is itself an ellipsoid and can be described by $\mathcal{E}^{\mathrm{o}}=T^{\prime-1}\left(B_{l_{2}^{n}}\right)$, where $T^{\prime}$ is the map dual to $T$.

We can now give the main geometric result, which is Theorem 4.3 of this paper:

Let $\alpha>\frac{1}{2}$. Then given any $n \in \mathbf{N}$ and any ball $B$ in $\mathbf{R}^{n}$, there is an associated ellipsoid $\mathcal{E}$ such that, for each $k, l \in \mathbf{N}$ with $k+l \leq n+1$, there are elements $x_{i}, a_{i}, y_{i}, b_{i} \in \mathbf{R}^{n}$ $\left(1 \leq i \leq 2^{k-1}\right)$ and subspaces $M, \tilde{M}, N, \tilde{N}$ of $\mathbf{R}^{n}$ with $\operatorname{codim}(M)<l, \operatorname{codim}(\tilde{M})<$ 
$l, \operatorname{dim}(N)<l$ and $\operatorname{dim}(\tilde{N})<l$, such that

(i) $B \cap M \subset \bigcup_{i=1}^{2^{k-1}}\left\{x_{i}+C(\alpha)(n /(k+l-1))^{\alpha} \mathcal{E}\right\}$,

(ii) $B^{\mathrm{o}} \cap \tilde{M} \subset \bigcup_{i=1}^{2^{k-1}}\left\{a_{i}+C(\alpha)(n /(k+l-1))^{\alpha} \mathcal{E}^{\mathrm{o}}\right\}$

and

(iii) $\mathcal{E} \subset \bigcup_{i=1}^{2^{k-1}}\left\{y_{i}+N+C(\alpha)(n /(k+l-1))^{\alpha} B\right\}$,

(iv) $\mathcal{E}^{\mathrm{o}} \subset \bigcup_{i=1}^{2^{k-1}}\left\{b_{i}+\tilde{N}+C(\alpha)(n /(k+l-1))^{\alpha} B^{\mathrm{o}}\right\}$.

Here $C(\alpha)$ is a positive constant depending only on $\alpha$.

As consequences of this result we derive the generalizations of the Santalo and inverse Santalo inequalities which are given in Theorems 5.1 and 5.2, respectively. The generalized Santalo inequality is as follows:

There is a universal constant $C \geq 1$ such that for any $n \in \mathbf{N}$ and any ball $B$ in $\mathbf{R}^{n}$ there is an associated ellipsoid $\mathcal{E}$ in $\mathbf{R}^{n}$ such that for any $m \in \mathbf{N}, 1 \leq m \leq n$, there are $m$-dimensional subspaces $M$ and $\tilde{M}$ of $\mathbf{R}^{n}$ with the property that

$$
\left(\frac{\operatorname{vol}_{m}(B \cap M) \operatorname{vol}_{m}\left(B^{\mathrm{o}} \cap \tilde{M}\right)}{\operatorname{vol}_{m}(\mathcal{E} \cap M) \operatorname{vol}_{m}\left(\mathcal{E}^{\mathrm{o}} \cap \tilde{M}\right)}\right)^{1 / m} \leq C .
$$

Here $\operatorname{vol}_{m}$ indicates m-dimensional Lebesgue measure.

The generalized inverse Santalo inequality reads:

There is a universal constant $C \geq 1$ such that for any $n \in \mathbf{N}$ and any ball $B$ in $\mathbf{R}^{n}$ there $i$ an associated ellipsoid $\mathcal{E}$ in $\mathbf{R}^{n}$ such that for any $l \in \mathbf{N} \cup\{0\}, l \leq n-1$, there are l-dimensional subspaces $N, \tilde{N}$ of $\mathbf{R}^{n}$ with the volume property that

$$
\left(\frac{\operatorname{vol}_{n}(\{N+C B\} \cap \mathcal{E}) \operatorname{vol}_{n}\left(\left\{\tilde{N}+C B^{\mathrm{o}}\right\} \cap \mathcal{E}^{\mathrm{o}}\right)}{\left(\operatorname{vol}_{n}\left(B_{l_{2}^{n}}\right)\right)^{2}}\right)^{1 /(n-l)} \geq \frac{1}{4}
$$

When $l=0$, this becomes the inverse Santalo inequality due to Bourgain and Milman.

In this direction we also mention some geometric results from Section 6 (Corollaries 6.7-6.9):

There is a universal constant $C \geq 1$ and for each ball $B$ in $\mathbf{R}^{n}$ and each pair $(k, l) \in \mathbf{N} \times \mathbf{N}$ with $1 \leq l \leq n$ there is an l-dimensional subspace $M \subset \mathbf{R}^{n}$ such that the section $M \cap B$ 
can be covered by $2^{k-1}$ Euclidean balls of radius $\rho$ with

$$
\begin{aligned}
\rho \leq & \left(C \text { Average }_{\varepsilon_{i}= \pm 1} \sup _{\left(t_{1}, \ldots, t_{n}\right) \in B}\left|\sum_{i=1}^{n} \varepsilon_{i} t_{i}\right|^{2}\right)^{1 / 2} \\
& \times\left(\log \left(\frac{n}{n+k-l}+1\right)\right)^{1 / 2}(n+k-l)^{-1 / 2} .
\end{aligned}
$$

The corresponding estimate for $\rho$ in the special case where $B=\operatorname{aconv}\left(x_{1}, \ldots, x_{n}\right)$ is the absolutely convex hull of $n$ points $x_{1}, \ldots, x_{n} \in \mathbf{R}^{n}$ and has non-empty interior is given by

$$
\rho \leq C \max _{1 \leq i \leq n}\left\|x_{i}\right\|_{2}(n+k-l)^{-1 / 2} \log ^{1 / 2}\left(\frac{n}{n+k-l}+1\right) .
$$

From this result we obtain, in the case $k=l$, the following volume estimate:

$$
\left(\frac{\operatorname{vol}_{m}(M \cap B)}{\operatorname{vol}_{m}\left(B_{l_{2}^{m}}\right)}\right)^{1 / m} \leq C n^{-1 / 2} \max _{1 \leq i \leq n}\left\|x_{i}\right\|_{2},
$$

where $\|\cdot\|_{2}$ is the Euclidean norm.

These geometric results follow from inequalities which are established between the so-called "covering numbers" of operators acting between Banach spaces and the Gelfand and Kolmogorov numbers of these operators. These covering numbers were introduced in [7] and are to be thought of as quantities "intermediate" between entropy numbers and Gelfand or Kolmogorov numbers. We illustrate the usefulness of these inequalities by combining them with a striking result of Pisier [13] to give new insight into coverings of convex sets and sections of convex sets in $\mathbf{R}^{n}$ by balls and cylinder sets. See [1] and [5] for other covering results. The combination of geometric and functional analysis may well be a powerful tool for the discovery of new geometric connections. However, the results are also of independent interest in geometric and functional analysis.

\section{Geometric Quantities and Operators between Banach Spaces}

Given any index set $X$, we denote by $l_{1}(X)$ the Banach space of all summable number families $\left(\xi_{t}\right)_{t \in X}$ over $X$, with norm given by

$$
\left\|\left(\xi_{t}\right)\right\|_{1}=\sum_{t \in X}\left|\xi_{t}\right|
$$

The dual of $l_{1}(X)$ is the Banach space $l_{\infty}(X)$ of all bounded number families $\left(\xi_{t}\right)_{t \in X}$ with the norm

$$
\left\|\left(\xi_{t}\right)\right\|_{\infty}=\sup _{t \in X}\left|\xi_{t}\right| .
$$

The closed unit ball in a Banach space $E$ will be denoted by $B_{E}$, and the value of a continuous linear functional $a \in E^{\prime}$, the dual of $E$, at $t \in E$ will be denoted by $\langle t, a\rangle$. 
Let $(X, d)$ be a metric space and let $B\left(x_{0}, \varepsilon\right)$ be the closed ball in $X$ with centre $x_{0}$ and radius $\varepsilon>0$. The entropy numbers $\varepsilon_{k}(K)(k \in \mathbf{N})$ of a bounded set $K \subset X$ are defined by taking $\varepsilon_{k}(K)$ to be the infimum of all those $\varepsilon>0$ such that there exist $k$ points $x_{1}, \ldots, x_{k}$ in $X$ such that

$$
K \subset \bigcup_{j=1}^{k} B\left(x_{j}, \varepsilon\right)
$$

Now let $T: E \rightarrow F$ be an operator (that is, a bounded linear map) from a Banach space $E$ to a Banach space $F$. The entropy numbers $\varepsilon_{k}(T)(k \in \mathbf{N})$ of $T$ are given by

$$
\varepsilon_{k}(T)=\varepsilon_{k}\left(T\left(B_{E}\right)\right) .
$$

Geometrically, one might think of $T\left(B_{E}\right)$ as being some kind of ellipsoid which is being covered by $k$ balls of the same radius: the idea is to take the infimum of all radii which achieve this. The dyadic entropy numbers $e_{n}(T)$ of $T$ are defined by

$$
e_{n}(T)=\varepsilon_{2^{n-1}}(T)
$$

for each $n \in \mathbf{N}$. It turns out that the dyadic entropy numbers are often more convenient to use than the entropy numbers. In addition to these numbers we shall need the covering numbers. The $(k, l)$ th $c$-covering number $c_{k, l}(T)(k, l \in \mathbf{N})$ of $T$ is defined (see [6]) to be the infimum of all those $\varepsilon>0$ such that there exists a subspace $M$ of $E$ with $\operatorname{codim} M<l$ and elements $y_{1}, \ldots, y_{2^{k-1}} \in F$ for which

$$
T\left(B_{E} \cap M\right) \subset \bigcup_{j=1}^{2^{k-1}}\left\{y_{j}+\varepsilon B_{F}\right\} .
$$

Given a bounded subset $K$ of $E$, the $(k, l)$ th $d$-covering number $d_{k, l}(K)$ of $K(k, l \in \mathbf{N})$ is defined to be the infimum of all those $\varepsilon>0$ such that there exists a subspace $N$ of $E$, with $\operatorname{dim} N<l$, and elements $x_{1}, \ldots, x_{2^{k-1}} \in E$ for which

$$
K \subset N+\bigcup_{j=1}^{2^{k-1}}\left\{x_{j}+\varepsilon B_{E}\right\}=\bigcup_{j=1}^{2^{k-1}}\left\{x_{j}+N+\varepsilon B_{E}\right\}
$$

The $d$-covering numbers $d_{k, l}(T)$ of an operator $T: E \rightarrow F$ are given by

$$
d_{k, l}(T)=d_{k, l}\left(T\left(B_{E}\right)\right) \text {. }
$$

These quantities may be expressed in terms of dyadic entropy numbers:

$$
c_{k, l}(T)=\inf \left\{e_{k}\left(T J_{M}^{E}\right): M \subset E, \operatorname{codim} M<l\right\}
$$

and

$$
d_{k, l}(T)=\inf \left\{e_{k}\left(Q_{N}^{F} T\right): N \subset F, \operatorname{dim} N<l\right\},
$$


where $J_{M}^{E}: M \rightarrow E$ is the natural embedding from $M$ into $E$ and $Q_{N}^{F}: F \rightarrow F / N$ is the quotient map from $F$ onto $F / N$. Note also that when $k=1$ the covering numbers reduce to more familiar quantities:

$$
c_{1, l}(T)=c_{l}(T), \quad \text { the } l \text { th Gelfand number of } T,
$$

and

$$
d_{1, l}(T)=d_{l}(T), \quad \text { the } l \text { th Kolmogorov number (or } l \text {-width) of } T .
$$

Moreover, when $l=1$ we recover the dyadic entropy numbers of $T$ :

$$
c_{k, 1}(T)=d_{k, 1}(T)=e_{k}(T) .
$$

We may therefore regard the $c$-covering (respectively, $d$-covering) numbers as being intermediate between entropy numbers and Gelfand (respectively, Kolmogorov) numbers.

Let $s_{k, l}$ denote either $c_{k, l}$ or $d_{k, l}$. It is easy to verify that the following additive and multiplicative properties hold:

$$
s_{k_{1}+k_{2}-1, l_{1}+l_{2}-1}(S+T) \leq s_{k_{1}, l_{1}}(S)+s_{k_{2}, l_{2}}(T)
$$

for all operators $S, T: E \rightarrow F$, and

$$
s_{k_{1}+k_{2}-1, l_{1}+l_{2}-1}(T S) \leq s_{k_{1}, l_{1}}(T) s_{k_{2}, l_{2}}(S)
$$

for all operators $S: E \rightarrow F$ and $T: F \rightarrow G$. Here $k_{1}, k_{2}, l_{1}$ and $l_{2}$ are arbitrary natural numbers. Moreover, if $\operatorname{dim} E=n$ and $I_{E}$ is the identity operator on $E$, then

$$
s_{k, l}\left(I_{E}\right) \leq 4 \cdot 2^{-(k-1) /(n-l+1)} \quad(\text { real case })
$$

and

$$
s_{k, l}\left(I_{E}\right) \leq 4 \cdot 2^{-(k-1) / 2(n-l+1)} \quad \text { (complex case) }
$$

for all $k \in \mathbf{N}$ and all $l \in \mathbf{N}$ with $l \leq n$. To establish these it is sufficient to deal with the real case and to observe that

$$
e_{k}\left(Q_{N}^{E}\right) \leq e_{k}\left(I_{E / N}\right) \leq 2^{2-(k-1) /(n-l+1)} \quad \text { for } \quad \operatorname{dim} N=l-1
$$

and

$$
e_{k}\left(J_{M}^{E}\right) \leq e_{k}\left(I_{M}\right) \leq 2^{2-(k-1) /(n-l+1)} \quad \text { for } \quad \operatorname{codim} M=k-l+1
$$

(see p. 21 of [8]). Note also that the $d$-covering numbers are surjective; that is, given any metric surjection $Q: E_{0} \rightarrow E$, which means that the open unit ball of $E_{0}$ is mapped by $Q$ onto the open unit ball of $E$, we have from the definition of covering numbers

$$
d_{k, l}(T Q)=d_{k, l}(T) .
$$

The $c$-covering numbers are injective (up to a factor 2); that is,

$$
c_{k, l}(J T) \leq c_{k, l}(T) \leq 2 c_{k, l}(J T)
$$


for any metric injection $J: F \rightarrow F_{0}$ (that is, with $\left\|J x\left|F_{0}\|=\| x\right| F\right\|$ for all $x \in F$ ). The factor 2 on the right-hand side of this last inequality arises from the fact that the centres of the $\varepsilon$-balls of the covering in $F_{0}$ need not have origins in $F$. However, to manage a covering with the centres of the balls in $F$ it is sufficient to enlarge the $\varepsilon$-balls of the covering in $F_{0}$ by the factor 2 .

We shall also need the approximation numbers $a_{k}(T)(k \in \mathbf{N})$ of $T$. These are defined by

$$
\begin{aligned}
a_{k}(T) & =\inf \{\|T-A\|: \operatorname{rank} A<k\} \\
& =\inf \left\{\varepsilon \geq 0: \text { there is } A, \operatorname{rank}(A)<k,(T-A)\left(B_{E}\right) \subset \varepsilon B_{F}\right\} .
\end{aligned}
$$

It is known (see Theorems 2.3.1 and 2.2.1 of [8]) that, for each $k \in \mathbf{N}$,

$$
c_{k}(T)=a_{k}\left(J_{F} T\right) \quad \text { and } \quad d_{k}(T)=a_{k}\left(T Q_{E}\right),
$$

where $J_{F}$ is the canonical injection from $F$ into $l_{\infty}\left(B_{F^{\prime}}\right)$, that is, $J_{F} x:=(\langle x, a\rangle)_{a \in B_{F^{\prime}}}$, and $Q_{E}$ is the canonical surjection from $l_{1}\left(B_{E}\right)$ onto $E, Q_{E}\left(\xi_{x}\right):=\sum_{x \in B_{E}} \xi_{x} x$.

\section{Inequalities Concerning Covering Numbers}

The main result states that the approximation numbers dominate the $c$ - and $d$-covering numbers in the sense of the following theorem, which is a generalization of the corresponding inequality in [4], relating entropy and approximation numbers. This theorem, or more precisely the version given by Corollary 3.5, is the bridge leading to the geometrical statements provided in Sections 4-6. It is also important for diverse applications in geometric and functional analysis.

Theorem 3.1. Let $E$ and $F$ be Banach spaces, let $T: E \rightarrow F$ be an operator and let $\alpha$ be a positive real number. Then

$$
\sup _{1 \leq k \leq n} k^{\alpha} \max \left\{c_{k, 2 l-1}(T), d_{k, 2 l-1}(T)\right\} \leq c(\alpha) \sup _{1 \leq k \leq n} k^{\alpha} a_{k+l-1}(T)
$$

for all $l, n \in \mathbf{N}$, where

$$
c(\alpha)=2^{7}(16(2+\alpha))^{\alpha} \quad \text { in the real case }
$$

and

$$
c(\alpha)=2^{7}(32(2+\alpha))^{\alpha} \text { in the complex case. }
$$

Proof. This follows the lines of that of Theorem 3.1.1 of [8], but we give the details for convenience. First we consider natural numbers $n$ of the form

$$
n=2^{N}, \quad N \in \mathbf{N},
$$

and let $l \in \mathbf{N}$. By the definition of the approximation numbers $a_{k}(T)$, there are operators $A_{j}: E \rightarrow F$ with $\operatorname{rank}\left(A_{j}\right)<2^{j}+l-1$ and

$$
\left\|T-A_{j}\right\| \leq 2 a_{2^{j}+l-1}(T) \quad \text { for } \quad j=0,1, \ldots, N,
$$


where $A_{0}=0$. Represent $T$ in the form

$$
T=\sum_{j=1}^{N}\left(A_{j}-A_{j-1}\right)+\left(T-A_{N}\right)
$$

and observe that

$$
\operatorname{rank}\left(A_{j}-A_{j-1}\right)<2^{j+1}+2 l-1 \quad \text { for } \quad j=1, \ldots, N .
$$

Use of the additivity of the covering numbers for fixed second index shows that

$$
d_{n_{1}+\cdots+n_{N}-(N-1), 2 l-1}(T) \leq \sum_{j=1}^{N} d_{n_{j}, 2 l-1}\left(A_{j}-A_{j-1}\right)+\left\|T-A_{N}\right\|
$$

for natural numbers $n_{j}$ to be chosen later. With $F_{j}:=\left(A_{j}-A_{j-1}\right)(E)$ we obtain

$$
d_{n_{j}, 2 l-1}\left(A_{j}-A_{j-1}\right) \leq\left\|A_{j}-A_{j-1}\right\| d_{n_{j}, 2 l-1}\left(I_{F_{j}}\right) .
$$

Since $\operatorname{dim} F_{j}<2^{j+1}+2 l-1$, we conclude from (2.3) that in the real case,

$$
d_{n_{j}, 2 l-1}\left(A_{j}-A_{j-1}\right) \leq 4 \cdot 2^{-\left(n_{j}-1\right) / 2^{j+1}}\left\|A_{j}-A_{j-1}\right\| .
$$

On the other hand,

$$
\left\|A_{j}-A_{j-1}\right\| \leq\left\|A_{j}-T\right\|+\left\|T-A_{j-1}\right\| \leq 4 a_{2^{j-1}+l-1}(T)
$$

for $j=1, \ldots, N$. Hence

$$
d_{n_{j}, 2 l-1}\left(A_{j}-A_{j-1}\right) \leq 2^{4} \cdot 2^{-\left(n_{j}-1\right) / 2^{j+1}} a_{2^{j-1}+l-1}(T)
$$

and so

$$
\begin{aligned}
d_{n_{1}+\cdots+n_{N}-(N-1), 2 l-1}(T) \leq & 2^{4} \sum_{j=1}^{N} 2^{-\left(n_{j}-1\right) / 2^{j+1}} a_{2^{j-1}+l-1}(T)+2 a_{2^{N}+l-1}(T) \\
\leq & \left(\sum_{j=1}^{N} 2^{4-\left(n_{j}-1\right) 2^{-j-1}-\alpha(j-1)}+2^{5-\alpha N}\right) \\
& \cdot \sup _{1 \leq j \leq 2^{N}} j^{\alpha} a_{j+l-1}(T) .
\end{aligned}
$$

Now we choose $K \in \mathbf{N}$ with

$$
1+\alpha \leq K \leq 2+\alpha
$$

and put

$$
n_{j}:=1+K(N-j) 2^{j+1}, \quad j=1, \ldots, N
$$

We then have

$$
2^{-\left(n_{j}-1\right) / 2^{j+1}}=2^{-K(N-j)}
$$


and

$$
\sum_{j=1}^{N} 2^{-K(N-j)-\alpha(j-1)} \leq 2^{2+\alpha-\alpha N}
$$

since $1 \leq K-\alpha \leq 2$. It follows that

$$
d_{n_{1}+\cdots+n_{N}-(N-1), 2 l-1}(T) \leq 2^{7+\alpha-\alpha N} \sup _{1 \leq j \leq N} j^{\alpha} a_{j+l-1}(T) .
$$

As for the subscript $n_{1}+\cdots+n_{N}-(N-1)$, observe that it equals

$$
K \sum_{j=1}^{N}(N-j) 2^{j+1}+1=4 K\left(2^{N}-N-1\right)+1
$$

which is bounded from above by $4 K 2^{N}$. Hence

$$
d_{2^{N+2} K, 2 l-1}(T) \leq 2^{7+\alpha-\alpha N} \sup _{1 \leq j \leq N} j^{\alpha} a_{j+l-1}(T), \quad N \in \mathbf{N} .
$$

Finally, we estimate $d_{n, 2 l-1}(T)$ for any $n \in \mathbf{N}$. First suppose that $n \geq 8 K$ and choose $N \geq 1$ so that

$$
8 K 2^{N-1} \leq n \leq 8 K 2^{N}
$$

Then

$$
d_{n, 2 l-1}(T) \leq d_{2^{N+2} K, 2 l-1}(T) \leq 2^{7+\alpha}(8 K)^{\alpha} n^{-\alpha} \sup _{1 \leq j \leq n} j^{\alpha} a_{j+l-1}(T),
$$

that is,

$$
n^{\alpha} d_{n, 2 l-1}(T) \leq 2^{7}(1+K)^{\alpha} \sup _{1 \leq j \leq n} j^{\alpha} a_{j+l-1}(T) .
$$

This estimate also holds when $1 \leq n \leq 8 K$. In fact, since

$$
d_{n, 2 l-1}(T) \leq d_{1, l}(T)=d_{l}(T) \leq a_{l}(T)
$$

we have

$$
\begin{aligned}
n^{\alpha} d_{n, 2 l-1}(T) & \leq(8 K)^{\alpha} a_{l}(T) \\
& \leq 2^{7}(16 K)^{\alpha} \sup _{1 \leq j \leq n} j^{\alpha} a_{j+l-1}(T)
\end{aligned}
$$

Since $K \leq 2+\alpha$ we obtain, for all $l, n \in \mathbf{N}$,

$$
n^{\alpha} d_{n, 2 l-1}(T) \leq 2^{7}(16(2+\alpha))^{\alpha} \sup _{1 \leq j \leq n} j^{\alpha} a_{j+l-1}(T) .
$$

This gives the result claimed for the $d$-covering numbers in the theorem in the real case. The complex case is handled in exactly the same way, using (2.4) instead of (2.3). The inequality for the $c$-covering numbers is established by proceeding along similar lines: we omit the details. The proof of the theorem is complete. 


\section{Geometrical Interpretation of Theorem 3.1}

Let $\alpha>0$ and let $\tilde{c}>c(\alpha)$, where $c(\alpha)$ is the constant of Theorem 3.1. Then the following holds:

Given any $n \in \mathbf{N}$ and any operator $T: E \rightarrow F$ between arbitrary Banach spaces $E$ and $F$, then for each $l \in \mathbf{N}$ and each $k \in \mathbf{N}$ with $1 \leq k \leq n$ there are elements $y_{i}, z_{i} \in F$ $\left(1 \leq i \leq 2^{k-1}\right)$ and subspaces $M \subset E$ and $N \subset F$ with $\operatorname{codim}(M)<2 l-1$ and $\operatorname{dim}(N)<2 l-1$, respectively, such that

$$
T\left(B_{E} \cap M\right) \subset \bigcup_{i=1}^{2^{k-1}}\left\{y_{i}+\rho B_{F}\right\}
$$

and

$$
T\left(B_{E}\right) \subset \bigcup_{i=1}^{2^{k-1}}\left\{z_{i}+N+\rho B_{F}\right\},
$$

where the radius $\rho$ satisfies

$$
\rho \leq k^{-\alpha} \sup _{1 \leq j \leq n} j^{\alpha} a_{j+l-1}(T)
$$

Corollary 3.2. With the same notation as in Theorem 3.1 and under the same conditions,

$$
\sup _{1 \leq k \leq n} k^{\alpha} d_{k, 2 l-1}(T) \leq c(\alpha) \sup _{1 \leq k \leq n} k^{\alpha} d_{k+l-1}(T)
$$

and

$$
\sup _{1 \leq k \leq n} k^{\alpha} c_{k, 2 l-1}(T) \leq 2 c(\alpha) \sup _{1 \leq k \leq n} k^{\alpha} c_{k+l-1}(T)
$$

for all $l, n \in \mathbf{N}$.

Proof. This follows immediately from Theorem 3.1 since

$$
d_{n, k}\left(T Q_{E}\right)=d_{n, k}(T) \text { and } a_{n}\left(T Q_{E}\right)=d_{n}(T),
$$

while also $c_{n, k}(T) \leq 2 c_{n, k}\left(J_{F} T\right)$ and $a_{n}\left(J_{F} T\right)=c_{n}(T)$.

When $l=1$ the inequality (3.1) of Theorem 3.1 becomes the inequality between entropy and approximation numbers which was proved in [4] (see also [11]). To formulate this special version of (3.1) we need the so-called symmetrized approximation numbers defined by

$$
t_{n}(T):=a_{n}\left(J_{F} T Q_{E}\right) .
$$

We recall that $J_{F}: F \rightarrow l_{\infty}\left(B_{F^{\prime}}\right)$ is the canonical injection and $Q_{E}: l_{1}\left(B_{E}\right) \rightarrow E$ is the canonical surjection. For these numbers we have (see p. 83 of [8]) for all $n \in \mathbf{N}$,

$$
t_{n}(T)=t_{n}\left(T^{\prime}\right)
$$


where $T^{\prime}$ is the dual of $T$, and

$$
t_{n}(T) \leq \min \left\{c_{n}(T), d_{n}(T)\right\}
$$

Since the entropy numbers are surjective and injective (up to a factor of 2; see pp. 12-13 of [8]), the following corollary (see [4] and p. 101 of [8]) is a direct consequence of Theorem 3.1.

Corollary 3.3. Let $\alpha$ be a positive real number and let $T: E \rightarrow F$ be an operator from a Banach space $E$ to a Banach space $F$. Then, for all $n \in \mathbf{N}$,

$$
\sup _{1 \leq k \leq n} k^{\alpha} \max \left\{e_{k}(T), e_{k}\left(T^{\prime}\right)\right\} \leq 2 c(\alpha) \sup _{1 \leq k \leq n} k^{\alpha} t_{k}(T)
$$

where $c(\alpha)$ is the constant from Theorem 3.1.

A generalization of the previous result may be given with the aid of the $b$-covering numbers of an operator $T: E \rightarrow F$, defined by

$$
b_{k, l}(T):=\max \left\{d_{k, l}(T), c_{k, l}\left(T^{\prime}\right)\right\}
$$

for $k, l \in \mathbf{N}$. These numbers possess the additivity and multiplicativity properties (2.1) and (2.2). Moreover, since

$$
d_{l}(T)=c_{l}\left(T^{\prime}\right), \quad d_{l}\left(T^{\prime}\right)=c_{l}(T)
$$

when $T$ is compact, and

$$
c_{l}\left(T^{\prime}\right) \leq d_{l}(T) \leq 5 c_{l}\left(T^{\prime}\right), \quad d_{l}\left(T^{\prime}\right)=c_{l}(T)
$$

for an arbitrary operator $T$ (see pp. 81-82 of [8]), we have

$$
b_{1, l}(T)=d_{l}(T), \quad b_{1, l}\left(T^{\prime}\right)=c_{l}(T) .
$$

Using these duality relations and the surjectivity of the $d$-covering numbers as well as the injectivity of the $c$-covering numbers (up to a factor of 2) we obtain from Theorem 3.1 and (2.5) the following:

Corollary 3.4. Let $\alpha$ be a positive real number, let $E$ and $F$ be Banach spaces and let $T: E \rightarrow F$ be an arbitrary operator. Then, for all $l, n \in \mathbf{N}$,

$$
\sup _{1 \leq k \leq n} k^{\alpha} b_{k, 2 l-1}(T) \leq 2 c(\alpha) \sup _{1 \leq k \leq n} k^{\alpha} d_{k+l-1}(T)
$$

and

$$
\sup _{1 \leq k \leq n} k^{\alpha} b_{k, 2 l-1}\left(T^{\prime}\right) \leq 2 c(\alpha) \sup _{1 \leq k \leq n} k^{\alpha} c_{k+l-1}(T),
$$

where $c(\alpha)$ is the constant of Theorem 3.1. 
In connection with Pisier's theorem given in the next section the following quantities will be useful. Let $E$ and $F$ be isomorphic Banach spaces and let $T: E \rightarrow F$ be a (bounded linear) isomorphism from $E$ onto $F$. The $\delta$-numbers of $T$ are defined by

$$
\delta_{k, l}(T):=\max \left\{b_{k, l}(T), b_{k, l}\left(T^{\prime-1}\right)\right\} \quad \text { for } \quad k, l \in \mathbf{N} .
$$

In view of the duality relations

$$
\delta_{1, l}(T)=\max \left\{d_{l}(T), c_{l}\left(T^{-1}\right)\right\}, \quad l \in \mathbf{N},
$$

from Corollary 3.4 we immediately obtain the following:

Corollary 3.5. Let $\alpha$ be a positive real number and let $T: E \rightarrow F$ be an isomorphism from a Banach space E onto a Banach space $F$. Then, for all $l, n \in \mathbf{N}$,

$$
\sup _{1 \leq k \leq n} k^{\alpha} \delta_{k, 2 l-1}(T) \leq 2 c(\alpha) \sup _{1 \leq k \leq n} k^{\alpha} \delta_{1, k+l+1}(T),
$$

where $c(\alpha)$ is the constant from Theorem 3.1.

To conclude this section we remark that the reason for the introduction of the covering numbers in [6] was that they are closely related to the eigenvalues of operators acting in Banach spaces. Indeed, for an operator $T: E \rightarrow E$ on a complex Banach space $E$ we consider the so-called covering moduli (see [6]) $g_{n, l}(T)$ of $T$ :

$$
g_{n, l}(T):=\inf _{k \in \mathbf{N}} 2^{(k-1) / 2 n} d_{k, l}(T) \quad(l, n \in \mathbf{N}) .
$$

A main result in [6] is the refinement of the classical spectral radius formula by means of these geometric parameters:

$$
\lim _{N \rightarrow \infty} g_{n, l}^{1 / N}\left(T^{N}\right)=\left(\prod_{j=1}^{n}\left|\lambda_{j+l-1}(T)\right|\right)^{1 / n} \quad(l, n \in \mathbf{N}) .
$$

Here $\left(\lambda_{k}(T)\right)$ is the sequence of eigenvalues of $T$ arranged in the order of nonincreasing absolute values greater than the essential spectral radius $r_{\text {ess }}(T):=\sup \{|\lambda|: \lambda$ belongs to the essential spectrum of $T\}$ of $T$, and repeated according to algebraic multiplicity. If $T$ has only $m(<\infty)$ distinct eigenvalues $\lambda$ with $|\lambda|>r_{\text {ess }}(T)$ and $M$ is the sum of their algebraic multiplicities, we put $\lambda_{k}(T)=r_{\text {ess }}(T)$ for all $k>M$. When $l=1$ we recover the spectral radius formula of Makai and Zemánek (see [6]); when $n=1$ we have the formula of König [9] for the Kolmogorov numbers. In the "intermediate" case the shift of length $l$ of the $n$th geometric mean of eigenvalues $\left(\prod_{j=1}^{n}\left|\lambda_{j+l-1}(T)\right|\right)^{1 / n}$ can be characterized by the "intermediate" quantity $\lim _{N \rightarrow \infty} g_{n, l}^{1 / N}\left(T^{N}\right)$.

\section{On a Theorem of Pisier}

In the geometry of Banach spaces an important part is played by Pisier's theorem [13] about the existence of certain isomorphisms from the $n$-dimensional Hilbert space $l_{2}^{n}$ onto an $n$-dimensional Banach space. This states the following. 
Theorem 4.1. For each real number $\alpha>\frac{1}{2}$ there is a constant $b(\alpha)$ such that, for any n-dimensional $(n \in \mathbf{N})$ real or complex Banach space E, there is an isomorphism $T: l_{2}^{n} \rightarrow$ E such that, for $l=1, \ldots, n$,

$$
\delta_{1, l}(T):=\max \left\{d_{l}(T), c_{l}\left(T^{-1}\right)\right\} \leq b(\alpha)(n / l)^{\alpha} .
$$

Moreover, the constant $b(\alpha)$ is of order $\left(\alpha-\frac{1}{2}\right)^{-1 / 2}$ when $\alpha \rightarrow \frac{1}{2}$.

We note that the conclusion of the theorem does not hold when $\alpha<\frac{1}{2}$, and that the case $\alpha=\frac{1}{2}$ remains open (see [13]). Using the inequality in Corollary 3.5, Pisier's theorem may be transformed into the following statement.

Theorem 4.2. Let $\alpha>\frac{1}{2}$. Then there is a constant $C(\alpha)$ such that for any n-dimensional $(n \in \mathbf{N})$ real or complex Banach space $E$ there is an isomorphism $T: l_{2}^{n} \rightarrow E$ such that

$$
\delta_{k, l}(T) \leq C(\alpha)\left(\frac{n}{k+l-1}\right)^{\alpha}
$$

for $k+l \leq n+1$. Moreover, the constant $C(\alpha)$ is of order $\left(\alpha-\frac{1}{2}\right)^{-1 / 2}$ when $\alpha \rightarrow \frac{1}{2}$.

Proof. Consideration of Corollary 3.5 and Theorem 4.1 gives

$$
\begin{aligned}
k^{\alpha} \delta_{k, 2 l-1}(T) & \leq 2 c(\alpha) \sup _{1 \leq j \leq k} j^{\alpha} \delta_{1, j+l-1}(T) \\
& \leq 2 c(\alpha) b(\alpha) \sup _{1 \leq j \leq k} j^{\alpha}\left(\frac{n}{j+l-1}\right)^{\alpha} \\
& \leq 2 c(\alpha) b(\alpha)\left(\frac{k}{k+l-1}\right)^{\alpha} n^{\alpha}
\end{aligned}
$$

Hence

$$
\delta_{k, 2 l-1}(T) \leq 2 c(\alpha) b(\alpha)\left(\frac{n}{k+l-1}\right)^{\alpha} .
$$

Changing from $2 l-1$ to $l$ and using the monotonicity of the $b_{k, l}$ with respect to $l$, we see that

$$
\delta_{k, l}(T) \leq 2^{\alpha+1} c(\alpha) b(\alpha)\left(\frac{n}{k+l-1}\right)^{\alpha} .
$$

This gives the desired estimate with the constant

$$
C(\alpha)=2^{\alpha+1} c(\alpha) b(\alpha)
$$

which is of order $\left(\alpha-\frac{1}{2}\right)^{-1 / 2}$ when $\alpha \rightarrow \frac{1}{2}$ since $b(\alpha)$ is of this order and $c(\alpha)$ is bounded as $\alpha \rightarrow \frac{1}{2}$. 
This last theorem has a nice geometrical interpretation in $\mathbf{R}^{n}$. Before giving this, we recall some notions from the geometry of convex sets in $\mathbf{R}^{n}$. A convex symmetric compact subset $B$ of $\mathbf{R}^{n}$ with the origin as an interior point will be called a ball. Given such a ball $B$, the function $\|\cdot\|_{B}$ given by

$$
\|x\|_{B}=\inf \{\lambda>0: x \in \lambda B\}
$$

is a norm on $\mathbf{R}^{n}$ such that

$$
B=\left\{x \in \mathbf{R}^{n}:\|x\|_{B} \leq 1\right\} .
$$

In this way we obtain a one-to-one correspondence between norms and balls. If $K$ is a subset of $\mathbf{R}^{n}$ its polar $K^{\mathrm{o}}$ is defined by

$$
K^{\mathrm{o}}=\left\{a \in \mathbf{R}^{n}:\langle x, a\rangle \leq 1 \text { for all } x \in K\right\}
$$

If $B$ is a ball, then $B^{\mathrm{o}}$ is also a ball and the norm corresponding to it is given by

$$
\|a\|_{B^{\circ}}=\sup _{x \in B}|\langle x, a\rangle|, \quad a \in \mathbf{R}^{n}
$$

which is just the norm dual to $\|\cdot\|_{B}$. Moreover, we use the term "ellipsoid" to mean any ball of the form $\mathcal{E}=T\left(B_{l_{2}^{n}}\right)$, where $T$ is an isomorphism on $\mathbf{R}^{n}$ and $B_{l_{2}^{n}}$ is the Euclidean ball of $\mathbf{R}^{n}$. The norm associated to $\mathcal{E}$ is plainly given by

$$
\|x\|_{\mathcal{E}}=\left\|T^{-1} x\right\|_{l_{2}^{n}}, \quad x \in \mathbf{R}^{n} .
$$

Furthermore, the polar $\mathcal{E}^{\mathrm{o}}$ of $\mathcal{E}$ is again an ellipsoid and can be described by $\mathcal{E}^{\mathrm{o}}=$ $T^{\prime-1}\left(B_{l_{2}^{n}}\right)$; the corresponding norm is

$$
\|a\|_{\mathcal{E}^{\circ}}=\left\|T^{\prime} a\right\|_{l_{2}^{n}} \quad \text { for } \quad a \in \mathbf{R}^{n} .
$$

We can now give a geometric consequence of Theorem 4.2.

Theorem 4.3. Let $\alpha>\frac{1}{2}$. Then given any $n \in \mathbf{N}$ and any ball $B$ in $\mathbf{R}^{n}$, there is an associated ellipsoid $\mathcal{E}$ such that, for each $k, l \in \mathbf{N}$ with $k+l \leq n+1$, there are elements $x_{i}, a_{i}, y_{i}, b_{i} \in \mathbf{R}^{n}\left(1 \leq i \leq 2^{k-1}\right)$ and subspaces $M, \tilde{M}, N, \tilde{N}$ of $\mathbf{R}^{n}$ with $\operatorname{codim}(M)<l, \operatorname{codim}(\tilde{M})<l, \operatorname{dim}(N)<l$ and $\operatorname{dim}(\tilde{N})<l$ such that

(i) $B \cap M \subset \bigcup_{i=1}^{2^{k-1}}\left\{x_{i}+C(\alpha)(n /(k+l-1))^{\alpha} \mathcal{E}\right\}$,

(ii) $B^{\mathrm{o}} \cap \tilde{M} \subset \bigcup_{i=1}^{2^{k-1}}\left\{a_{i}+C(\alpha)(n /(k+l-1))^{\alpha} \mathcal{E}^{\mathrm{o}}\right\}$

and

(iii) $\mathcal{E} \subset \bigcup_{i=1}^{2^{k-1}}\left\{y_{i}+N+C(\alpha)(n /(k+l-1))^{\alpha} B\right\}$,

(iv) $\mathcal{E}^{\circ} \subset \bigcup_{i=1}^{2^{k-1}}\left\{b_{i}+\tilde{N}+C(\alpha)(n /(k+l-1))^{\alpha} B^{o}\right\}$.

Here $C(\alpha)$ is the constant from Theorem 4.2. 
Proof. Let $E$ be the Banach space $\mathbf{R}^{n}$ with $B$ as its unit ball. By Theorem 4.2 there is an isomorphism $T: l_{2}^{n} \rightarrow E$ such that

$$
\delta_{k, l}(T) \leq C(\alpha)\left(\frac{n}{k+l-1}\right)^{\alpha} .
$$

The ellipsoid $\mathcal{E}=T\left(B_{l_{2}^{n}}\right)$ satisfies the conclusions of the theorem. Indeed, (i) and (ii) follow from

$$
\max \left\{c_{k, l}\left(T^{-1}\right), c_{k, l}\left(T^{\prime}\right)\right\} \leq \delta_{k, l}(T),
$$

while (iii) and (iv) result from

$$
\begin{aligned}
& \max \left\{d_{k, l}(T), d_{k, l}\left(T^{\prime-1}\right)\right\} \leq \delta_{k, l}(T), \\
& \max \left\{d_{k, l}(T), d_{k, l}\left(T^{\prime-1}\right)\right\} \leq \delta_{k, l}(T) .
\end{aligned}
$$

Remark. The case $k=1$ is just the result of Pisier stated above as Theorem 4.1. Theorem 4.3 complements results of König and Milman (see [10]).

\section{Volume Inequalities of Santalo Type}

We derive two results on volumes of sections of balls in $\mathbf{R}^{n}$. In a certain sense these generalize the Santalo inequality [14] and the inverse Santalo inequality due to Bourgain and Milman [3] (see [13]). The first theorem can be regarded as a generalization of the Santalo inequality.

Theorem 5.1. There is a universal constant $C \geq 1$ such that for any $n \in \mathbf{N}$ and any ball $B$ in $\mathbf{R}^{n}$ there is an associated ellipsoid $\mathcal{E}$ in $\mathbf{R}^{n}$ such that for any $m \in \mathbf{N}, 1 \leq m \leq n$, there are $m$-dimensional subspaces $M$ and $\tilde{M}$ of $\mathbf{R}^{n}$ with the property that

$$
\left(\frac{\operatorname{vol}_{m}(B \cap M) \operatorname{vol}_{m}\left(B^{\mathrm{o}} \cap \tilde{M}\right)}{\operatorname{vol}_{m}(\mathcal{E} \cap M) \operatorname{vol}_{m}\left(\mathcal{E}^{\mathrm{o}} \cap \tilde{M}\right)}\right)^{1 / m} \leq C .
$$

Here $\operatorname{vol}_{m}$ indicates m-dimensional Lebesgue measure.

Proof. We use Theorem 4.3(i) with $\alpha=1$ and $k-1=n-l=m$, and conclude that

$$
B \cap M \subset \bigcup_{i=1}^{2^{m}} S\left(x_{i}\right),
$$

where $S\left(x_{i}\right)=\left\{x_{i}+B(1) \mathcal{E}\right\} \cap M$ and $\operatorname{dim} M=m$. By comparison of volumes we obtain

$$
\operatorname{vol}_{m}(B \cap M) \leq \sum_{i=1}^{2^{m}} \operatorname{vol}_{m}\left(S\left(x_{i}\right)\right)
$$


Since

$$
\operatorname{vol}_{m}\left(S\left(x_{i}\right)\right)=\operatorname{vol}_{m}\left(S\left(-x_{i}\right)\right)
$$

we obtain, by the Brunn-Minkowski inequality,

$$
\begin{aligned}
\operatorname{vol}_{m}\left(S\left(x_{i}\right)\right) & =\left(\frac{1}{2} \operatorname{vol}_{m}^{1 / m}\left(S\left(x_{i}\right)\right)+\frac{1}{2} \operatorname{vol}_{m}^{1 / m}\left(S\left(-x_{i}\right)\right)\right)^{m} \\
& \leq \operatorname{vol}_{m}\left(\frac{1}{2} S\left(x_{i}\right)+\frac{1}{2} S\left(-x_{i}\right)\right) \\
& \leq \operatorname{vol}_{m}((C(1) \mathcal{E}) \cap M) \\
& \leq \operatorname{vol}_{m}(C(1)(\mathcal{E} \cap M)) \\
& \leq C(1)^{m} \operatorname{vol}_{m}(\mathcal{E} \cap M) .
\end{aligned}
$$

Hence

$$
\operatorname{vol}_{m}(B \cap M) \leq 2^{m} C(1)^{m} \operatorname{vol}_{m}(\mathcal{E} \cap M)
$$

and so

$$
\left(\frac{\operatorname{vol}_{m}(B \cap M)}{\operatorname{vol}_{m}(\mathcal{E} \cap M)}\right)^{1 / m} \leq 2 C(1)
$$

Analogously, from Theorem 4.3(ii) we obtain

$$
\left(\frac{\operatorname{vol}_{m}\left(B^{\mathrm{o}} \cap \tilde{M}\right)}{\operatorname{vol}_{m}\left(\mathcal{E}^{\mathrm{o}} \cap \tilde{M}\right)}\right)^{1 / m} \leq 2 C(1)
$$

Combination of the last two inequalities gives the desired result, with $C=$ $4(C(1))^{2}$.

When $m=n$ the inequality (5.1) becomes the well-known Santalo inequality

$$
\left(\frac{\operatorname{vol}_{n}(B) \operatorname{vol}_{n}\left(B^{\mathrm{o}}\right)}{\left(\operatorname{vol}_{n}\left(B_{l_{2}^{n}}\right)\right)^{2}}\right)^{1 / n} \leq C
$$

since $\operatorname{vol}_{n}(\mathcal{E}) \operatorname{vol}_{n}\left(\mathcal{E}^{\mathrm{o}}\right)=\left(\operatorname{vol}_{n}\left(B_{l_{2}^{n}}\right)\right)^{2}$. Santalo proved this inequality with $C=1$.

By proceeding along lines of proof similar to those of Theorem 5.1 we obtain from (iii) and (iv) of Theorem 4.3 the following generalization of the inverse Santalo inequality due to Bourgain and Milman [3] (see [13]).

Theorem 5.2. There is a universal constant $C \geq 1$ such that for any $n \in \mathbf{N}$ and any ball $B$ in $\mathbf{R}^{n}$ there is an associated ellipsoid $\mathcal{E}$ in $\mathbf{R}^{n}$ such that for anyl $l \in \mathbf{N} \cup\{0\}, l \leq n-1$, there are l-dimensional subspaces $N, \tilde{N}$ of $\mathbf{R}^{n}$ with the property that

$$
\left(\frac{\operatorname{vol}_{n}(\{N+C B\} \cap \mathcal{E}) \operatorname{vol}_{n}\left(\left\{\tilde{N}+C B^{\mathrm{o}}\right\} \cap \mathcal{E}^{\mathrm{o}}\right)}{\left(\operatorname{vol}_{n}\left(B_{l_{2}^{n}}\right)\right)^{2}}\right)^{1 /(n-l)} \geq \frac{1}{4}
$$


When $l=0$, this becomes the inverse Santalo inequality due to Bourgain and Milman:

$$
\left(\frac{\operatorname{vol}_{n}(B) \operatorname{vol}_{n}\left(B^{\mathrm{o}}\right)}{\left(\operatorname{vol}_{n}\left(B_{l_{2}^{n}}\right)\right)^{2}}\right)^{1 / n} \geq \frac{1}{4 C^{2}}
$$

For more on volume inequalities we refer to the book by Pisier [13]

Remark. The Santalo and inverse Santalo inequalities have interesting applications in discrete computational geometry: see, for example, [2]. The generalizations of these inequalities given in Theorems 5.1 and 5.2 should yield additional information in the spirit of [2].

\section{6. $s$-Covering Numbers, Gaussian and Rademacher Averages}

This section is devoted to some applications of the inequalities in Section 3. We derive inequalities between $c$ - and $d$-covering numbers and Gaussian or Rademacher averages which generalize the famous inequalities of Sudakov and Pajor and Tomczak-Jaegermann [12]. For this purpose we recall the definition of the so-called Gaussian average or $l$-norm of an operator $T$ from $l_{2}^{n}$ into a (real) Banach space $E$. The $l$-norm $l(T)$ of $T$ is defined by

$$
l(T)=\left(\int_{\mathbf{R}^{n}}\|T x\|^{2} d \gamma_{n}(x)\right)^{1 / 2},
$$

where $\gamma_{n}$ denotes the canonical (normalized) Gaussian measure on the Euclidean space $\mathbf{R}^{n}$. Moreover, for any operator $T$ from $l_{2}$ into $E$ we define $l(T)$ by

$$
l(T)=\sup \left\{l(T A): A \in L\left(l_{2}^{n}, l_{2}\right),\|A\| \leq 1, n \in \mathbf{N}\right\} .
$$

We use a minoration of $l(T)$ proved by Pajor and Tomczak-Jaegermann [12] in the following theorem.

Theorem 6.1. Let E be a Banach space. Then

$$
\sup _{k \in \mathbf{N}} k^{1 / 2} d_{k}(T) \leq C l(T)
$$

for every compact operator $T \in L\left(l_{2}, E\right)$, and

$$
\sup _{k \in \mathbf{N}} k^{1 / 2} c_{k}(T) \leq C l\left(T^{\prime}\right)
$$

for every compact operator $T \in L\left(E, l_{2}\right)$, where $C(\geq 1)$ is a universal constant.

Combining (6.1) and (6.2) with the inequalities (3.5) and (3.6) in Corollary 3.4 we obtain the following minoration theorem for $c$ - and $d$-covering numbers. 
Theorem 6.2. Let E be a Banach space. Then

$$
\sup _{k, l \in \mathbf{N}}(k+l-1)^{1 / 2} d_{k, l}(T) \leq C l(T)
$$

for every compact operator $T \in L\left(l_{2}, E\right)$, and

$$
\sup _{k, l \in \mathbf{N}}(k+l-1)^{1 / 2} c_{k, l}(T) \leq C l\left(T^{\prime}\right)
$$

for every compact operator $T \in L\left(E, l_{2}\right)$, where $C(\geq 1)$ is a universal constant.

The inequalities (6.3) and (6.4) include the famous Sudakov minoration theorem [15] if we fix $l=1$. Theorem 6.2 may be considered as a minoration theorem intermediate between those of Sudakov and Pajor and Tomczak-Jaegermann.

Next we derive inequalities for Rademacher averages instead of Gaussian averages. For this purpose let $\varphi=\left\{f_{1}, \ldots, f_{m}\right\}$ be an orthonormal basis of $l_{2}^{m}$ and let $T \in L\left(l_{2}^{m}, E\right)$. The Rademacher average $r_{\varphi}(T)$ of $T$ with respect to $\varphi$ is given by

$$
r_{\varphi}(T)=\left(\text { Average }_{\varepsilon_{i}= \pm 1}\left\|\sum_{i=1}^{m} \varepsilon_{i} T\left(f_{i}\right)\right\|^{2}\right)^{1 / 2}
$$

It is well known that $r_{\varphi}(T) \leq l(T) c$ for some universal constant $c$, independent of the particular choice of $\varphi$. The following minoration theorem for the Rademacher averages was proved in [7].

Theorem 6.3. Let $E$ be a Banach space and let $m \in \mathbf{N}$. Then, for any orthonormal basis $\varphi$ of $l_{2}^{m}$,

$$
\sup _{1 \leq k \leq n, k \in \mathbf{N}}\left(\log \left(\frac{n}{k}+1\right)\right)^{-1 / 2} k^{1 / 2} d_{k}(T) \leq c r_{\varphi}(T)
$$

for every operator $T \in L\left(l_{2}^{m}, E\right)$ of rank $n \leq m$, and

$$
\sup _{1 \leq k \leq n, k \in \mathbf{N}}\left(\log \left(\frac{n}{k}+1\right)\right)^{-1 / 2} k^{1 / 2} c_{k}(T) \leq c r_{\varphi}\left(T^{\prime}\right)
$$

for every operator $T \in L\left(E, l_{2}^{m}\right)$ of rank $n \leq m$. Here $c(\geq 1)$ is a universal constant.

Now we state a corresponding theorem for $c$ - and $d$-covering numbers.

Theorem 6.4. Let $E$ be a Banach space and let $m \in \mathbf{N}$. Then, for every orthonormal basis $\varphi$ of $l_{2}^{m}$,

$$
\sup _{k, l \in \mathbf{N}}\left(\log \left(\frac{n}{k+l-1}+1\right)\right)^{-1 / 2}(k+l-1)^{1 / 2} d_{k, l}(T) \leq C r_{\varphi}(T)
$$


for every operator $T \in L\left(l_{2}^{m}, E\right)$ of rank $n \leq m$, and

$$
\sup _{k, l \in \mathbf{N}}\left(\log \left(\frac{n}{k+l-1}+1\right)\right)^{-1 / 2}(k+l-1)^{1 / 2} c_{k, l}(T) \leq C r_{\varphi}\left(T^{\prime}\right)
$$

for every operator $T \in L\left(E, l_{2}^{m}\right)$ of rank $n \leq m$. Here $C(\geq 1)$ is a universal constant.

Proof. We prove only (6.7) as the proof of (6.8) is similar. First we establish the inequality

$$
(k+l-1)^{\alpha} d_{k, 2 l-1}(T) \leq c(\alpha) \sup _{1 \leq j \leq k}(l+j-1)^{\alpha} d_{j+l-1}(T), \quad \alpha>0 .
$$

Indeed, from (3.5) of Corollary 3.4 we have

$$
\begin{aligned}
k^{\alpha} d_{k, 2 l-1}(T) & \leq c(\alpha) \sup _{1 \leq j \leq k} j^{\alpha} d_{j+l-1}(T) \\
& \leq c(\alpha) \sup _{1 \leq j \leq k}(j /(j+l-1))^{\alpha} \sup _{1 \leq j \leq k}(j+l-1)^{\alpha} d_{j+l-1}(T) \\
& \leq c(\alpha)(k /(k+l-1))^{\alpha} \sup _{1 \leq j \leq k}(j+l-1)^{\alpha} d_{j+l-1}(T) .
\end{aligned}
$$

This gives (6.9); here $c(\alpha)$ is a constant depending on $\alpha$. Moreover, from (6.5) we find that

$$
\begin{aligned}
\sup _{1 \leq j \leq k}(j+l-1) d_{j+l-1}(T) & \leq \sup _{1 \leq j \leq k}\left((j+l-1) \log \left(\frac{n}{j+l-1}+1\right)\right)^{1 / 2} c r_{\varphi}(T) \\
& \leq c(k+l-1)^{1 / 2}\left(\log \left(\frac{n}{k+l-1}+1\right)\right)^{1 / 2} r_{\varphi}(T)
\end{aligned}
$$

and consequently

$$
(k+l-1)^{1 / 2}\left(\log \left(\frac{n}{k+l-1}+1\right)\right)^{-1 / 2} d_{k+l-1}(T) \leq c r_{\varphi}(T) .
$$

Combining (6.10) and (6.9) with $\alpha=1$ we obtain

$$
\begin{aligned}
(k+l-1) d_{k, 2 l-1}(T) & \leq c(1) \sup _{1 \leq j \leq k}(l+j-1) d_{j+l-1}(T) \\
& \leq c(1) c(k+l-1)^{1 / 2}\left(\log \left(\frac{n}{k+l-1}+1\right)\right)^{1 / 2} r_{\varphi}(T),
\end{aligned}
$$

so that

$$
(k+j-1)^{1 / 2}(\log (n /(k+l-1)+1))^{-1 / 2} \leq c(1) c r_{\varphi}(T) .
$$

Furthermore, since $(k+2 l-2)^{1 / 2}(\log (n /(k+2 l-2)+1))^{-1 / 2}$ is bounded above by $2 \sqrt{2}$ times the corresponding expression with $2 l-2$ replaced by $l-1$, we conclude from (6.11) that

$$
(k+l-1)^{1 / 2}\left(\log \left(\frac{n}{k+l-1}+1\right)\right)^{-1 / 2} d_{k, 2 l-1}(T) \leq 2 \sqrt{2} c(1) c r_{\varphi}(T) .
$$

Since $d_{k, 2 l} \leq d_{k, 2 l-1}$ we can pass from $d_{k, 2 l-1}$ to $d_{k, l}$ and obtain (6.7). 
For operators $T \in L\left(l_{1}^{n}, l_{2}\right)$ or $T \in L\left(l_{2}, l_{\infty}^{n}\right)$ the following modification of Theorem 6.3 was proved in [7].

Theorem 6.5. There is a universal constant $C \geq 1$ such that

$$
\sup _{1 \leq k \leq n} k^{1 / 2}\left(\log \left(\frac{n}{k}+1\right)\right)^{-1 / 2} d_{k}(T) \leq C\|T\|
$$

for all $T \in L\left(l_{2}, l_{\infty}^{n}\right)$ and all $n \in \mathbf{N}$, and

$$
\sup _{1 \leq k \leq n} k^{1 / 2}\left(\log \left(\frac{n}{k}+1\right)\right)^{-1 / 2} c_{k}(T) \leq C\|T\|
$$

for all $T \in L\left(l_{1}^{n}, l_{2}\right)$ and all $n \in \mathbf{N}$.

The following counterpart of this theorem for $c$ - and $d$-covering numbers may be proved in a way similar to that used for Theorem 6.4.

Theorem 6.6. There is a universal constant $C \geq 1$ such that

$$
\sup _{k, l \in \mathbf{N}}(k+l-1)^{1 / 2}\left(\log \left(\frac{n}{k+l-1}+1\right)\right)^{-1 / 2} d_{k, l}(T) \leq C\|T\|
$$

for all $T \in L\left(l_{2}, l_{\infty}^{n}\right)$ and all $n \in \mathbf{N}$, and

$$
\sup _{k, l \in \mathbf{N}}(k+l-1)^{1 / 2}\left(\log \left(\frac{n}{k+l-1}+1\right)\right)^{-1 / 2} c_{k, l}(T) \leq C\|T\|
$$

for all $T \in L\left(l_{1}^{n}, l_{2}\right)$ and all $n \in \mathbf{N}$.

Now we turn to some geometrical considerations. Let $B \subset \mathbf{R}^{n}$ be a compact, convex and symmetric set with non-empty interior. Equipped with the Minkowski functional $\|\cdot\|_{B}$ of $B$ the vector space $\mathbf{R}^{n}$ becomes a normed space with $B$ as its unit ball. Application of (6.8) to the canonical isomorphism

$$
T:\left(\mathbf{R}^{n},\|\cdot\|_{B}\right) \rightarrow\left(\mathbf{R}^{n},\|\cdot\|_{2}\right)=l_{2}^{n}
$$

gives the following corollary.

Corollary 6.7. There is a universal constant $C \geq 1$ and for each pair $(k, l) \in \mathbf{N} \times \mathbf{N}$ with $1 \leq l \leq n$ there is an l-dimensional subspace $M \subset \mathbf{R}^{n}$ such that the $k$ th dyadic entropy number $e_{k}(M \cap B)$ of the intersection $M \cap B$ satisfies

$$
\begin{aligned}
e_{k}(M \cap B) \leq & \left(C \text { Average }_{\varepsilon_{i}= \pm 1} \sup _{\left(t_{1}, \ldots, t_{n}\right) \in B}\left|\sum_{i=1}^{n} \varepsilon_{i} t_{i}\right|^{2}\right)^{1 / 2} \\
& \times\left(\log \left(\frac{n}{n+k-l}+1\right)\right)^{1 / 2}(n+k-l)^{-1 / 2} .
\end{aligned}
$$


This means that $M \cap B$ is covered by $2^{k-1}$ Euclidean balls of radius $\rho$, where $\rho$ is bounded by the right-hand side of (6.17). In particular, for each $l, 1 \leq l \leq n$, there is an l-dimensional subspace $M \subset \mathbf{R}^{n}$ such that

$$
e_{l}(M \cap B) \leq C\left(\text { Average }_{\varepsilon_{i}= \pm 1} \sup _{\left(t_{1}, \ldots, t_{n}\right) \in B}\left|\sum_{i=1}^{n} \varepsilon_{i} t_{i}\right|^{2}\right)^{1 / 2} n^{-1 / 2} .
$$

The corresponding geometrical interpretation of the inequality (6.16) of Theorem 6.6 is as follows.

Corollary 6.8. Let $x_{1}, \ldots, x_{n} \in \mathbf{R}^{n}$ be vectors such that their absolutely convex hull $B=\operatorname{aconv}\left(x_{1}, \ldots, x_{n}\right)$ has non-empty interior. Then for each pair $(k, l)$ with $k \in \mathbf{N}, 1 \leq$ $l \leq n$, there is an l-dimensional subspace $M \subset \mathbf{R}^{n}$ such that

$$
e_{k}(M \cap B) \leq C \max _{1 \leq i \leq n}\left\|x_{i}\right\|_{2}(n+k-l)^{-1 / 2} \log ^{1 / 2}\left(\frac{n}{n+k-l}+1\right),
$$

where $C \geq 1$ is a universal constant. In particular, for $k=l$ we have

$$
e_{l}(M \cap B) \leq C n^{-1 / 2} \max _{1 \leq i \leq n}\left\|x_{i}\right\|_{2} .
$$

From (6.20) the following volume inequality is immediate.

Corollary 6.9. Let $x_{1}, \ldots, x_{n} \in \mathbf{R}^{n}$ be such that their absolutely convex hull $B=$ $\operatorname{aconv}\left(x_{1}, \ldots, x_{n}\right)$ has non-empty interior. Then for each $m \in \mathbf{N}, 1 \leq m \leq n$, there is an $m$-dimensional subspace $M \subset \mathbf{R}^{n}$ such that the volume ratio inequality

$$
\left(\frac{\operatorname{vol}_{m}(M \cap B)}{\operatorname{vol}_{m}\left(B_{l_{2}^{m}}\right)}\right)^{1 / m} \leq C n^{-1 / 2} \max _{1 \leq i \leq n}\left\|x_{i}\right\|_{2}
$$

holds, where $C \geq 1$ is a universal constant.

\section{Acknowledgement}

We are indebted to the referees for their very helpful and constructive comments on the original version of the paper.

\section{References}

1. K. Ball and A. Pajor, The entropy of convex bodies with "few" extreme points, London Mathematical Society Lecture Notes Series, vol. 158, Cambridge University Press, Cambridge, 1990, pp. 25-32.

2. I. Bárány and Z. Füredi, Computing the volume is difficult, Discrete Comput. Geom. 2 (1987), 319-326.

3. J. Bourgain and V. D. Milman, New volume ratio properties for convex symmetric bodies in $\mathbf{R}^{n}$, Invent. Math. 88 (1987), 319-340. 
4. B. Carl, Entropy numbers, s-numbers and eigenvalue problems, J. Funct. Anal. 41 (1981), 290-306.

5. B. Carl, Metric entropy of convex hulls in Hilbert spaces, Bull. London Math. Soc. 29 (1997), 452-458.

6. B. Carl and Jr. Makai, New covering numbers and spectral properties of operators in Banach spaces, Anal. Math. 17 (1991), 183-209.

7. B. Carl and A. Pajor, Gelfand numbers of operators with values in Hilbert spaces, Invent. Math. 94 (1988), 459-504.

8. B. Carl and I. Stephani, Entropy, Compactness and the Approximation of Operators, Cambridge University Press, Cambridge, 1990.

9. H. König, A formula for the eigenvalues of a compact operator, Studia Math. 65 (1979), 141-146.

10. H. König and V. D. Milman, On the covering numbers of convex bodies. In Proc. Israel Functional Analysis Seminar GAFA, Lecture Notes in Mathematics, vol. 1267, Springer-Verlag, Berlin, 1987, pp. 82-95.

11. V. D. Milman and G. Pisier, Banach spaces with a weak cotype 2 property, Israel J. Math. 54 (1986), 139-158.

12. A. Pajor and N. Tomczak-Jaegermann, Subspaces of small codimension of finite-dimensional Banach spaces, Proc. Amer. Math. Soc. 97 (1986), 637-642.

13. G. Pisier, The Volume of Convex Bodies and Banach Space Geometry, Cambridge University Press, Cambridge, 1989.

14. L. A. Santalo, Un invariante afin pasa los cuerpos convexos del espacio de $n$ dimentiones, Portugal Math. 8 (1949), 155-161.

15. V. N. Sudakov, Gaussian processes and measures of solid angles in Hilbert space, Soviet Math. Dokl. 12 (1981), 412-415.

Received May 27, 1998, and in revised form January 25, 1999. 